\title{
A new method of winner determination for economic resource allocation in cloud computing systems
}

\author{
Ebrahim Behrouzian Nejad ${ }^{1,}{ }^{*}$, Rezvan Ali poorsabzevari ${ }^{2}$ \\ ${ }^{1}$ Department of Computer, Andimeshk Branch, Islamic Azad University, Andimeshk, Iran \\ ${ }^{2}$ Department of Computer, Khouzestan Science and Research Branch, Islamic Azad University, \\ Ahvaz, Iran
}

\author{
Index Terms \\ Cloud Computing \\ Economic Resource Allocation \\ Imperialist Competitive Algorithm \\ (ICA) \\ Winner Determination
}

Received: 19 October 2014

Accepted: 20 July 2015

Published: 22 February 2016

\begin{abstract}
Cloud computing systems are large-scale distributed systems so that they focus more on large-scale resource sharing, cooperation of several organizations, and their use in new applications. One of the main challenges in this realm is resource allocation. There are many different ways of resource allocation in cloud computing. One of the common methods of resource allocation is economic methods. Among these methods, the auctionbased method has greater prominence compared with the Fixed-Price method. The double combinatorial auction is one of the proper ways of resource allocation in cloud computing. This method includes two phases: winner determination and resource allocation. This paper presents a new method to determine the winner in double combinatorial auctionbased resource allocation using the Imperialist Competitive Algorithm (ICA). The experimental results show that the number of winner users is higher than the genetic algorithm in our new proposed method. On the other hand, in the proposed algorithm, the number of winner providers is higher in the genetic algorithm.
\end{abstract}

(C) 2016 TAF Publishing. All rights reserved.

\section{INTRODUCTION}

Cloud computing, often referred to as simply "the cloud," is the delivery of on-demand computing resources, everything from applications to data centers over the Internet on a pay-for-use basis. Cloud computing systems are large-scale distributed systems so that they focus more on large-scale resource sharing, cooperation of several organizations and their use in new applications. Achieving high performance in a cloud system requires effective resource allocation [1]. Resource allocation is one of the

\footnotetext{
*Corresponding author: Ebrahim Behrouzian Nejad E-mail: behrouzian.e@gmail.com
}

integral issues of cloud computing and data center management [2]. These days cloud providers use fixedprice mechanisms to allocate resources to their users. But these mechanisms don't provide efficient allocation and don't maximize the revenue of providers. Economic model of cloud-based resource allocations is more suitable to adjust supply and demand of resources. A better alternative for resource allocation is to use auction-based mechanisms. The most appropriate mechanism in cloud computing for resource allocation and pricing is combinatorial auction [3]. Prices should only depend on the demand and supply condition, in order to promote a fair exchange between providers (sellers) and users. Therefore, the double combinatorial auction to resource 
allocation in cloud computing is a proper model. Double combinatorial auction includes two steps. At first, winner determination is done from auction offers by solving an optimization model that aims at maximizing social welfare (by distinguishing between buyers' pay and total income of sellers); Second, the allocation and pricing of resources is done among a winners set. But this trend of auction has some problems. The winner determination is a NP-hard problem when the number of volunteers and resources is in a particular scale and the winner cannot be found in a polynomial time [4]. Therefore, the heuristic algorithms solve problem from a scientific perspective very well.

The rest of the paper is organized as follows: Section II includes basic concepts on cloud computing systems. In section III, the concept of resource allocation is presented. Section IV explains the ICA in detail. In section V, related works are presented. Our new proposed method is presented in section VI. Evaluation results are shown in section VII. Finally, conclusion and future work will be presented in section VIII.

\section{A. Could Computing}

In recent years cloud computing has begun to be regarded as animportant event in the information technology world. Cloud computing is a pervasive issue that tries to increase the capacity and capabilities of processing dynamically and reliably and to increase the quality assurance with no need of developing the existing infrastructure and purchasing new software or hiring more staff. Cloud computing is a new processing method for delivering information technology on the network. However, the main idea of cloud computing is not new. John McCarty in 1960, predicted that computing provides some tools to help public. It is obvious that the lack of defined standards in cloud computing has led to confusion. For this reason, the standardization of cloud computing definitions has been done recently. A large number of researchers have tried to define cloud computing in functional aspects, but they didn't succeed and there is not a comprehensive definition for it. Among different definitions, the definition of NIST is as follows: ( $($ cloud computing is a model that has been designed foreasy access to the configurable computing resources such as networks , servers and services, ... that is created with lowest management endeavour and by providers' participation )) .[5]

\section{B. Resource Allocation}

Resource allocation is one of the continuous and integral issues of cloud computing and data center management. Consider the following hypothetical scenario: a cloud service provider allocates servers to tenant virtual machines according to the requirement of CPU, memory and disk. At a later date, the provider improves model and allocates the network bandwidth resources to tenant virtual machines. At this stage, the strategy of resource allocation depends on some limitations such as servers' capacity and network band width of center. In fact, resource allocation includes dividing and allocating resources which have some special limitations such as server performance assurance, network efficiency and flout tolerance. Many of these resource allocation problems are NP-Hard problems. A large number of management tools of new data centersoperate through proper heuristical methods to solve independent problems. The recent network virtualization researches have used greedy heuristical methods to allocate virtual machines to data centers, for example. The resource allocation problems can be divided in two categories: Firstly, accepting new requests and brining virtual machines for them and putting them on hosts. Second: resource allocation process that optimizes previous allocations.

The first problem can be considered as a pack bin problem with different bin size. Common algorithms for pack bin problems include: worst fit, best fit, first fit and next fit [6].Double combinatorial auction is not of the most common combinatorial auction approaches that can model as an optimization problem to maximize social welfare (buyers' total pay and sellers' total income). It is possible that several buyers and several sellers volunteer for goods bid bundles. Participants in auction send a request about buying or selling a goods bid bundle. Any bundle is a buying request that all of its goods will be bought or a selling offer that all of its goods will be sold. In this auction the request is seal bid. It means that the participants in the market don't know each other and only the auctioneer has access to information [7].

\section{Imperialist Competitive Algorithm (ICA)}

In this section we describe Imperialist Competitive Algorithm (ICA). This algorithm is an evolutionary optimization method that is inspired by imperialistic competition. We customize it and use ICA to propose our new method in section VI.

ICA starts with an initial population [10]. Population individuals called countries are divided into two types: colonies and imperialists that all together form some empires. Imperialistic competition among these empires forms the basis of ICA. During this competition, weak empires collapse and powerful ones take possession of their colonies. Imperialistic competition hopefully converges to a state in which there is only one empire and 
its colonies are in the same position and have the same cost as the imperialist.

The steps of ICAare mentioned as below:

(1)Select some random points from the function and initialize the empires.

(2)Move the colonies towards their relevant imperialist

(Assimilation).

(3)Randomly change the position of some colonies

(Revolution).

(4) If there is a colony in an empire which has lower costthan the imperialist, exchange the positions of thatcolony and the imperialist.

(5) Unite the similar empires.

(6) Compute the total cost of all empires.

(7) Pick the weakest colony (colonies) from the weakest empires and give it (them) to one of the empires

(Imperialistic competition).

(8) Eliminate the powerless empires.

(9)If stop conditions are satisfied, then stop.If not, go to the

second point [10].

\section{Related Works}

Fuji Ware et al in [4] used double combinatorial action to resource allocation and provided current/future booking in the spot markets and front markets. They have used a combinatorial liner programming to determine winner of auction that led to the auction winner to be determined on a large scale and for a longer time. Schnizler et al in [8] used combinatorial auction in grid computing to allocate resources. In this paper, they offered combinatorial auction-based resource allocation protocol in which, a user can offer a price for any mix of needed resources to perform his/her duties. This protocol involves an approximation algorithm to solve the combinatorial auction problem. The proposed approach includes four steps: requesting information from local market auction, generating proposals, determining resource allocation and assigning tasks to winner resources. They have used an approximation algorithm to solve combinatorial auction problem that finds a near to optimization allocation and includes two steps. In first step, an approximation is determined by combinatorial auction liner programming and in the last step named hill climbing step, the quality algorithm improves the solution by performing the sequence of greedy allocation procedures obtained in the first step. The results showed that their proposed model has an economical effect on performance of system. Ghorbanzadeh et al in [9] have used genetic combinatorial algorithm to determine auction winner in grid. They have used two types of genetic combinatorial algorithm in order to improve the efficiency of genetic algorithm. The experimental results showed that the combinatorial algorithm of genetic hill climbing and simulated-annealing led to better results than genetic algorithm.

\section{PROPOSED METHOD}

Proposed algorithm to solve winner determination problem in double combinatorial auction in cloud computing is ICA. ICA identifies the auction winners. In fact, the output of this algorithm identifies that which one of the users and providers' bid bundles won the auction.

\section{E. Bid Bundles}

$\mathrm{B}=\left\{\mathrm{B}_{1}, \ldots, \mathrm{B}_{\mathrm{j}}, \ldots, \mathrm{B}_{\mathrm{n}}\right\}$ is a collection of bid bundles that includes $\mathrm{n}$ bundles ( $\mathrm{n}$ is the total number of users and providers). The Bj bid can include four tipple $\left(E_{j}, R_{j}, S_{j}, P_{j}\right.$ ), where : $E_{j}=\left(E_{1 j} \ldots E_{i j} \ldots E_{n j}\right):$ if $E_{i j}>0$ then it is the unit of requested ECU.IfE $E_{i j}<0$, then it is the unit of supplied ECU.

$R_{j}=\left(R_{1 j}, \ldots, R_{i j}, \ldots \ldots, R_{n j}\right)$ where: If $R_{i j}>0$ then it is the unit of requested memory. If $\mathrm{R}_{\mathrm{ij}}<0$, it is the unit of supplied memory.

$S_{j}=\left(S_{1 j}, \ldots ., S_{i j}, \ldots \ldots, S_{n j}\right)$ where: If $S_{j}>0$ then it is the unit of requested storage. If $S_{j}<0$, it is the unit of supplied storage.

$\mathrm{P}_{\mathrm{ij}} \in \mathrm{R}$ is the amount that the bidder is eager to pay. It is considered as a buying bid if $\mathrm{p}_{\mathrm{i} j}>0$ or it is considered as a selling bid if $\mathrm{p}_{\mathrm{ij}}<0$.

It should be noted that bundle will be won and all of its resources be allocated or never won. Moreover, every bid bundle of provider can cover several requests of users. It means that several requests can be provided by a single provider, but the resources of a provider can only be allocated to a single user.

\section{F. ICA for Winner Determination Problem}

As mentioned in other sections, ICA is one of the approaches of evolutionary computing that tries to find optimal answers of different optimization problems. This approach provides an algorithm for solving mathematical problems of optimization by mathematical modeling of socio-political evolution process [10]. As shown in (1), in this algorithm the number of dimensions of a country is equal to the number of bid bundles of users and providers.

COUNTRY $=\left[\mathrm{P}_{1}, \mathrm{P}_{2} \ldots \mathrm{P}_{\mathrm{NVAR}}\right]$

That Pi $\in\{0,1\}$ and 1 indicates that the bid bundle has been won and 0 indicates that the bid bundle has not been won. In fact, any country is an array that includes 0 and 1.The aim of this paper was to allocate economical 
resources so that in this allocation the profits of users and providers are considered. In fact, in double combinatorial auction, the provider that offers the lowest price will win and also the user with the highest proposed price will win.Therefore a country is better if the sum of its winner bid bundles is more than others.Therefore the cost function of this algorithm can be calculated by (2) and (3).

$\sum_{\mathrm{j}=1}^{\mathrm{n}} \mathrm{Pj} \mathrm{Xj}, \mathrm{X}_{\mathrm{j}} \in\{0,1\}$

$\sum$ ajxj $\leq 0, \forall \mathrm{aj}_{\mathrm{j}} \in\{\mathrm{Ej}, \mathrm{Rj}, \mathrm{Sj}\}$

$\mathrm{X}_{\mathrm{j}} \in\{0,1\}, \forall \mathrm{j} \in\{0,1, \ldots, \mathrm{n}\}$

Equation (2) means that a combination of winners and their offered price are higher than others considered as an answer. Of course the combination should fulfill users' requests. This control has been computedby (3) and it means that the answer with negative winner bundles is proper i.e. the prospered resources aremore than requested resources. $\mathrm{P}_{\mathrm{j}}$ is the offered price of bid bundle number $\mathrm{j}$ and $\mathrm{Xi}$ is the amount of $\mathrm{j}$-th member of array of country and indicates that whether this country won. In proposed algorithm, the following Assimilation was considered to determine the condition of new habitation in attraction policy: $\alpha=0.5, \beta=2$.

Every empire that could not add to its strength will fail in imperialist rivalries. This failing occurs gradually. It means that the weak empire lost his colony and stronger empire will seize these colonies gradually [10].For this purpose, firstly the total cost function of each empire and each imperial power is calculated by subtracting the cost of the weakest empire fromthe total cost function of each empire. After that, capture probability of each empire is calculated according to its power and by dividing the power of each empire by the sum of all imperial powers. Now, the empire withthe highest capture probability randomly selects a country from the weakest empire.

\section{EVALUATION RESULTS}

The JAVA programming language was used to simulate proposed algorithm. As it has been said before, the ICA was used to determine the winner of algorithm. The parameters of this algorithm are as follows:

The number of users' bid bundles: 600, the number of providers' bid bundles: 400, the number of problem dimensions: The number of users' bid bindles+ the number of providers' bid bundles, the number of countries: 100, the number of empires: 10 , the number of decades: 100 ,

Revolution Rates: $0.1, \beta=2, \alpha=0.5$.

In fact, the providers bid the characteristics of their virtual machines in their bid bundles for which Amazon EC2 Standard has been used. The virtual machine m3 includes different sizes of medium, large, $x$ large and $2 x$ large from which $\mathrm{x}$ large and $2 \mathrm{x}$ large are used. Users and providers bid the amount and prices of three resources in their bid bundles.Moreover it is supposed that each bid bundle includes request or offer resources for a time unit (hour) during whichdesired virtual machines had this amount. The data of these machines have been shown in Table I below:

TABLE I

THE CHARACTERISTICS OF VIRTUAL MACHINES [11]

\begin{tabular}{|c|c|c|c|c|}
\hline $\begin{array}{l}\text { Vm } \\
\text { type }\end{array}$ & $\begin{array}{c}\text { ECU } \\
\text { Insta } \\
\text { nce }\end{array}$ & $\begin{array}{c}\text { Memor } \\
\text { y (GB) }\end{array}$ & $\begin{array}{c}\text { Storage } \\
\text { (GB) }\end{array}$ & $\begin{array}{c}\text { Windo } \\
\text { ws } \\
\text { Usage }\end{array}$ \\
\hline $\begin{array}{c}\text { m3.xla } \\
\text { rge }\end{array}$ & 13 & 15 & $\begin{array}{c}2 \times 40 \\
\text { SSD }\end{array}$ & $\begin{array}{c}\$ 0.532 \\
\text { per } \\
\text { Hour }\end{array}$ \\
\hline $\begin{array}{c}\mathrm{m} 3.2 \mathrm{xl} \\
\text { arge }\end{array}$ & 26 & 30 & $\begin{array}{c}2 \times 80 \\
\text { SSD }\end{array}$ & $\begin{array}{c}\$ 1.064 \\
\text { per } \\
\text { Hour } \\
\end{array}$ \\
\hline
\end{tabular}

ECU is a technical term for processor. Many of providers have various ways to allocate and share processor, therefore it is difficult to comprise processor performance in deferent clouds. Therefore different providers have used different technical terms to define the processors of servers, such as ECU, VPC, CORES and so on.The ECU has been used in this kind of virtual machines.

The Proposed algorithm is compared with genetic algorithm from convergence, the number of users and the number of providers' point of view. The results of comparison are represented in following diagram:

The Fig. 1shows the convergence of genetic algorithms and winner determination in solving winner determination problem:

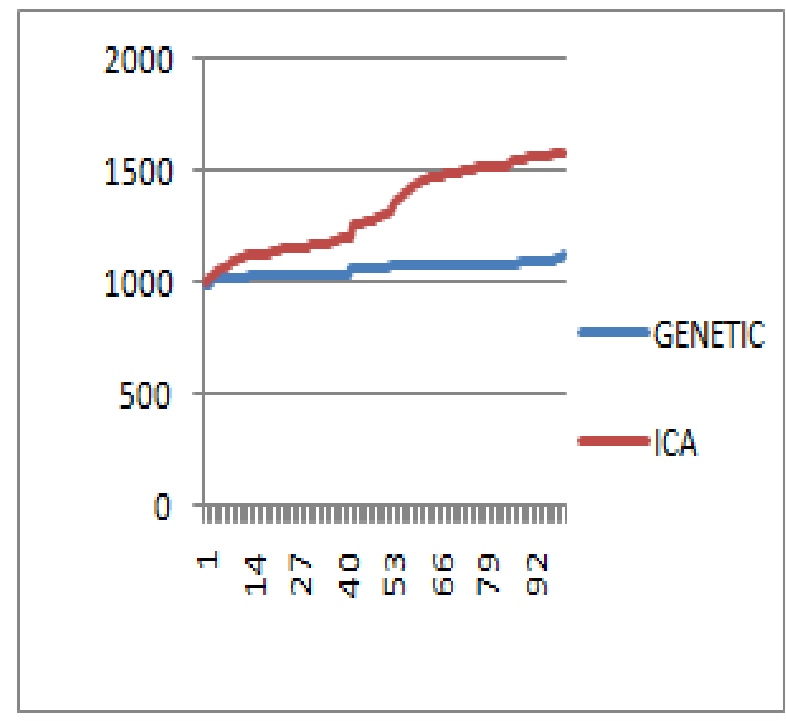

Fig. 1. The convergence diagram of algorithms 
The number of users' bid bundles is 600. As it can be seen in Fig 2, proposed algorithm has more winner users than genetic algorithm and worked $22.5 \%$ better than genetic algorithm.

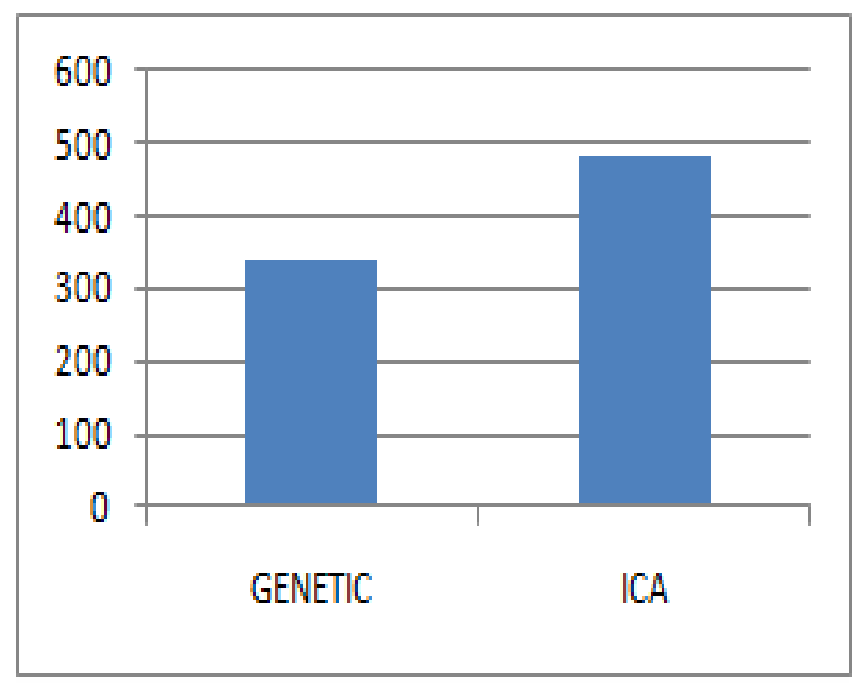

Fig. 2: Number of winner users of algorithms

Fig. 3 shows the number of winner providers in ICA and genetic algorithm. The number of providers' bid bundles is considered at 400.The results showed that the number of winner providers of genetic algorithm was higher than ICA and worked $4.5 \%$ better than ICA.

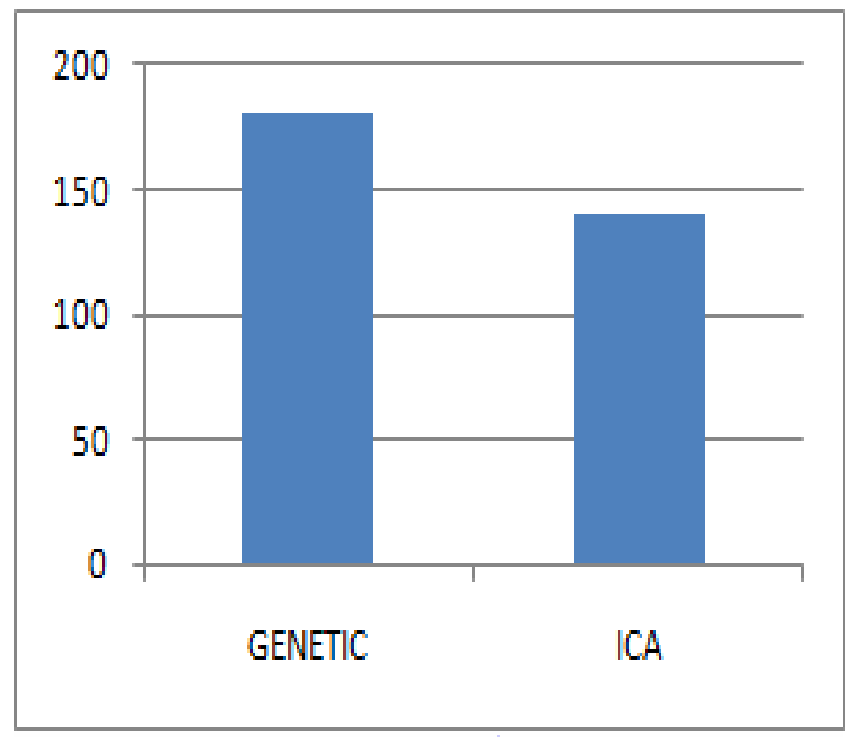

Fig. 3. The number of winner providers of algorithms

\section{CONCLUSION}

Achieving high performance in a cloud system requires effective resource allocation. This paper investigates the use of proposed algorithm forwinner determination in double combinatorial auction-based resource allocation in cloud computing. Resource allocation with auction-based method has some advantages compared with fixed-price method that current providers enjoy from these advantages in cloud computing. This algorithm leads to resource allocation performed according to the demandsupply condition. In each bid bundle, the total price of bid is suggested. The winner determination stage is done by ICA, after receiving the bid bundles. At this stage the winner user and provider are identified by taking account of the aim of problem that is user and provider profit.The experimental results show that in our new proposed method, the number of winner users is higher than that from the genetic algorithm. On the other hand, in proposed algorithm, the number of winner providers is higher in genetic algorithm.Its reason can be indicated by evaluating the convergence diagram of algorithms. The high amount of target function indicates the increase of winner users, because bided price of users is a positive number and bided price of providers is a negative number and our target function is given by the sum of bided prices of auction winners.

\section{REFERENCES}

[1] Q. Zhang, L. Cheng and R. Boutaba, "Cloud computing: State-of-the-art and research challenges," Journal of Internet Services and Applications, vol. 1, no. 1, pp. 718, 2010. DOI: 10.1007/s13174-010-0007-6

[2] A. Rai, R. Bhagwan and S. Guha, "Generalized resource allocation for the cloud," In proceedings of the Third ACM Symposium on Cloud Computing, 2012.

[3] S. Zaman and D. Grosu, "Combinatorial auction-based mechanisms for vm provisioning and allocation in clouds," In Cluster, Cloud and Grid Computing (CCGrid), 2012 12th IEEE/ACM International Symposium on, 2012. DOI: $10.1109 / C C G r i d .2012 .22$

[4] I. Fujiwara, K. Aida and I. Ono, "Applying double-sided combinational auctions to resource allocation in cloud computing," in Applications and the Internet (SAINT), 2010 10th IEEE/IPSJ International Symposium on, 2010. DOI: 10.1109 /SAINT.2010.93

[5] F. Teng, "Evidence and management scheduling of tasks on architectures distributed," Phd thesis, Appliqu'ees Mathematics and Computer, 2011.

[6] S. C. Tam, H. K. Tam, L. M. Tam and T. Zhang, "A new optimization method, the algorithm of changes, for bin packing problem," in Bio-Inspired Computing: Theories and Applications (BIC-TA), IEEE Fifth International Conference on, 2010.

DOI: 10.1109/BICTA.2010.5645122 
[7] M. Xia, J. Stallaert, and A. Whinston "Solving the combinatorial double auction problem," European Journal of Operational Research, vol. 164, no. 1, pp. 239-251, 2005. DOI: 10.1016/j.ejor.2003.11.018

[8] B. Schnizler, D. Neumann, D. Veit and C. Weinhardt. "Trading grid services a multi-attribute combinatorial approach," European Journal of Operational Research, vol. 187, no. 3, pp. 943-961, 2008. DOI: 10.1016/j.ejor.2006.05.049

[9] F. Gorbanzadeh and A. A. P. Kazem, "Hybrid genetic algorithms for solving winner determination problem in combinatorial double auction in grid," IAES International Journal of Artificial Intelligence, vol. 1, no. 2, pp. 54-62, 2012.

[10] E. A. Gargari and C. Lucas, "Imperialist competitive algorithm: An algorithm for optimization inspired by imperialistic competition," in Evolutionary Computation, 2007, IEEE Congress on (pp. 46614667). DOI: 10.1109/CEC.2007.4425083

[11] "Amazon Web Service site,". [Online]. Available: http://aws.amazon.com/ec2/instance-types

— This article does not have any appendix. - 